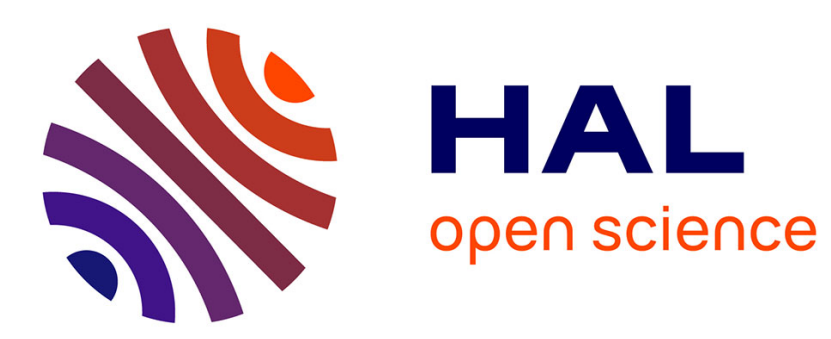

\title{
Joint Identification of Stepper Motor Parameters and of Initial Encoder Offset
}

\author{
Romain Delpoux, Marc Bodson, Thierry Floquet
}

\section{To cite this version:}

Romain Delpoux, Marc Bodson, Thierry Floquet. Joint Identification of Stepper Motor Parameters and of Initial Encoder Offset. 16th IFAC Symposium on System Identification, Jul 2012, Brussels,

Belgium. hal-00686351

\section{HAL Id: hal-00686351 \\ https://hal.inria.fr/hal-00686351}

Submitted on 10 Apr 2012

HAL is a multi-disciplinary open access archive for the deposit and dissemination of scientific research documents, whether they are published or not. The documents may come from teaching and research institutions in France or abroad, or from public or private research centers.
L'archive ouverte pluridisciplinaire HAL, est destinée au dépôt et à la diffusion de documents scientifiques de niveau recherche, publiés ou non, émanant des établissements d'enseignement et de recherche français ou étrangers, des laboratoires publics ou privés. 


\title{
Joint Identification of Stepper Motor Parameters and of Initial Encoder Offset *
}

\author{
R. Delpoux ${ }^{*, * *}$ M. Bodson ${ }^{* * *}$ T. Floquet ${ }^{*, * *}$ \\ ${ }^{*}$ LAGIS (CNRS FRE 3303), Ecole Centrale de Lille, 59651 Villeneuve \\ d'Ascq cedex, France (e-mail: romain.delpoux@ec-lille.fr, \\ thierry.floquet@ec-lille.fr). \\ ** Projet Non-A, INRIA, Lille Nord Europe, France \\ *** PEARL, Department of Electrical and Computeur Engineering, \\ University of Utah, Salt Lake City, UT 84112, USA (e-mail: \\ bodson@eng.utah.edu)
}

\begin{abstract}
The paper presents a new procedure to identify at the same time the electrical parameters of a permanent magnet stepper motor (PMSM) and the initial offset of an incremental encoder. The model considers effects due to the permanent magnet and to variable reluctance, and the resulting theory is applicable to cases where both or only one of these terms is present. The standard DQ model of PMSM's assumes that the permanent magnet is lined up with a winding when the position is zero. When an incremental encoder is used, an initialization procedure is required to zero the initial offset. In contrast, this paper computes a transformed model that accounts for the initial offset. then develops a least-squares identification algorithm that estimates the machine's electrical parameters together with the offset angle. Experiments show that the estimation procedure and a closed-control method using the estimated offset perform similarly compared to when the offset is reset to zero using an initilization procedure.
\end{abstract}

Keywords: Permanent magnet stepper motor, synchronous motors, parameter identification, offset calibration, sliding modes.

\section{INTRODUCTION}

Permanent Magnet Stepper Motors (PMSM's) are widely used in industry for position control, especially in manufacturing applications. PMSM's are more robust than brush DC motors and produce a high torque per volume. They are often controlled in open-loop, although the potential loss of synchronism limits operation away from resonances and from high acceleration profiles. These problems can be resolved by using closed-loop control methods with position sensors of sufficient precision. The typical position sensor in industrial applications is the incremental encoder. With such a sensor, the zero position is the motor position when the system is powered-up. However, precise control methods using the $D Q$ transformation require that the zero position be aligned with the permanent magnet. An initialization procedure must then be implemented to zero the initial position offset. Typically, this is achieved by applying a large current to one phase of the motor. However, this technique assumes that the load and friction torques are sufficiently small so that the position converges to the desired value. In this paper, we propose an identification procedure that eliminates the need for initialization of the position sensor. It combines the identification of the

\footnotetext{
* This work was supported by Ministry of Higher Education and Research, Nord-Pas-de-Calais Regional Council and FEDER through the "Contrat de Projets Etat Region (CPER) 2007-2013". Marc Bodson acknowledges the support of the École Centrale de Lille through a visiting professorship.
}

machine's electrical parameters with the estimation of the offset angle.

Modelling of PMSM's is discussed in Goeldel [1984] and Sinha et al. [1974], while the estimation of PMSM parameters was studied in Blauch et al. [1993], Kim and Lorenz [2002], and Nahid Mobarakeh et al. [2001]. Methods to calibrate the position sensor include Konghirun [2005], where an automatic offset calibration is proposed for Quadrature Encoder Pulse (QEP), Segarich et al. [2009] where a method based on the induced currents through the stator windings is developed, and Jung and Ha [1998] where a solution is proposed using the same transformation as in this article, but machine parameters are not estimated.

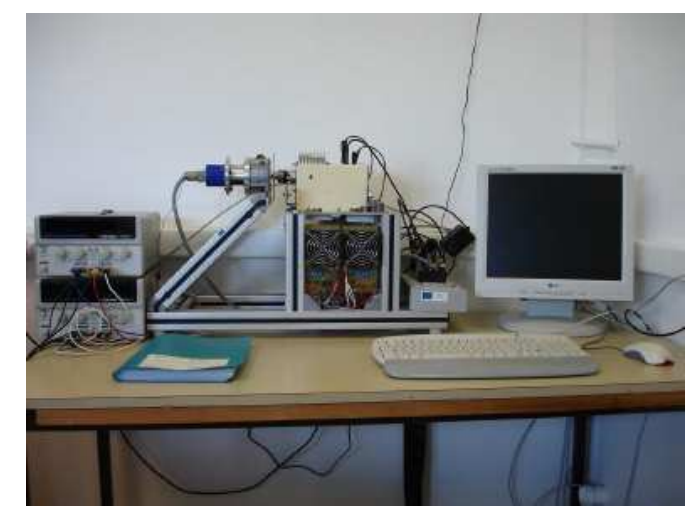

Fig. 1. Test Bench available at the LAGIS 


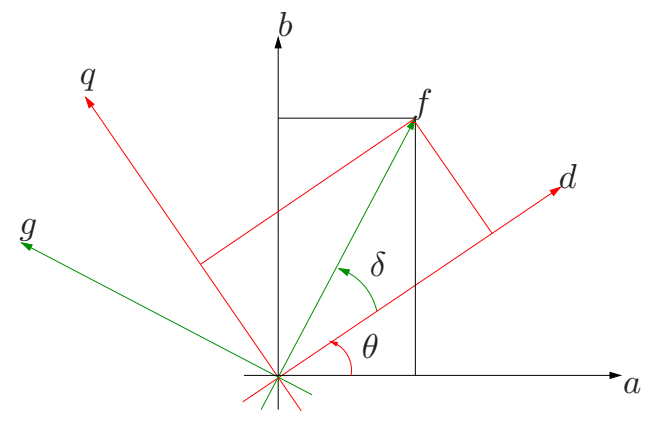

Fig. 2. Transformation of variables in the different frames

The contribution of this paper is to show that joint estimation of the motor parameters and of the offset is possible with a relatively simple algorithm. A model for a hybrid stepper motor is considered, which includes pure PM stepper motors and synchronous reluctance motors as special cases. Experiments were performed using the test bench shown on figure 1, available at the LAGIS laboratory, École Centrale de Lille in France. The results show that the method is as effective at obtaining the motor parameters as a method based on $D Q$ identification and encoder initialization. Closed-loop control results are also comparable.

The article is divided into four parts. Section 2 presents the model of the PMSM in three different reference frames. In Section 3, an identification procedure is developed for systems including an offset angle. Section 4 presents the experimental results of the identification and compares the results to those obtained when the sensor is initialized. The last section is devoted to evaluating the closed-loop performance.

\section{MODEL OF THE PMSM}

In this section, the model of a hybrid PMSM is presented in three different frames, including the frame that will be used for the joint identification of the motor parameters and of the position offset. The axes of the different frames are shown in Fig. 2.

\subsection{Model in the winding frame $a-b$ :}

The electrical equations for the two windings $a$ and $b$ of the PMSM are given by

$$
\left\{\begin{array}{l}
\frac{d \Psi_{a}}{d t}=v_{a}-R i_{a} \\
\frac{d \Psi_{b}}{d t}=v_{b}-R i_{b}
\end{array}\right.
$$

where $\Psi_{a}$ and $\Psi_{b}$ are the total flux linkages in windings $a$ and $b, v_{a}$ and $v_{b}$ are the voltages applied to the windings, $i_{a}$ and $i_{b}$ are the winding currents, and $R$ is the resistance of a winding. Considering a PMSM with $n_{p}$ rotor teeth, the total flux linkages are assumed to be of the form

$$
\left(\begin{array}{l}
\Psi_{a} \\
\Psi_{b}
\end{array}\right)=L(\theta)\left(\begin{array}{c}
i_{a} \\
i_{b}
\end{array}\right)+\Psi_{0} D(\theta)
$$

where

$$
\begin{aligned}
L(\theta) & =\left(\begin{array}{cc}
L_{0}+L_{2} \cos \left(2 n_{p} \theta\right) & L_{2} \sin \left(2 n_{p} \theta\right) \\
L_{2} \sin \left(2 n_{p} \theta\right) & L_{0}-L_{2} \cos \left(2 n_{p} \theta\right)
\end{array}\right) \\
D(\theta) & =\left(\begin{array}{c}
\cos \left(n_{p} \theta\right) \\
\sin \left(n_{p} \theta\right)
\end{array}\right)
\end{aligned}
$$

$\theta$ is the angular position of the rotor, $L_{0}$ is the average inductance of a winding as the motor rotates, $L_{2}$ is the mean-to-peak variation of the inductance, and $\Psi_{0}$ is the contribution of the permanent magnet to the flux $\Psi_{a}$ when $\theta=0$.

Using the expressions for the fluxes, the electrical equations become

$$
\begin{aligned}
L(\theta) \frac{d}{d t}\left(\begin{array}{c}
i_{a} \\
i_{b}
\end{array}\right)= & \left(\begin{array}{c}
v_{a}-R i_{a} \\
v_{b}-R i_{b}
\end{array}\right)-\frac{\partial L(\theta)}{\partial \theta} \omega\left(\begin{array}{c}
i_{a} \\
i_{b}
\end{array}\right) \\
& -\Psi_{0} \omega \frac{\partial D(\theta)}{\partial \theta}
\end{aligned}
$$

where $\omega=d \theta / d t$ is the angular velocity of the rotor and

$$
\begin{aligned}
& \frac{\partial L(\theta)}{\partial \theta}=2 n_{p} L_{2}\left(\begin{array}{ll}
-\sin \left(2 n_{p} \theta\right) & \cos \left(2 n_{p} \theta\right) \\
\cos \left(2 n_{p} \theta\right) & \sin \left(2 n_{p} \theta\right)
\end{array}\right) \\
& \frac{\partial D(\theta)}{\partial \theta}=n_{P}\left(\begin{array}{l}
-\sin \left(n_{p} \theta\right) \\
\cos \left(n_{p} \theta\right)
\end{array}\right)
\end{aligned}
$$

The mechanical equation is

$$
J \frac{d \omega}{d t}=-f_{v} \omega-C_{r} \operatorname{sgn}(\omega)+\tau_{e}
$$

where $J$ is the moment of inertia of the rotor (including the load), $f_{v}$ is the coefficient of viscous friction, $C_{r}$ is the coefficient of Coulomb friction and $\tau_{e}$ is the torque. By conservation of energy, the torque is given by

$$
\tau_{e}=\Psi_{0}\left(i_{a} i_{b}\right) \frac{\partial D(\theta)}{\partial \theta}+\frac{1}{2}\left(\begin{array}{ll}
i_{a} & i_{b}
\end{array}\right) \frac{\partial L(\theta)}{\partial \theta}\left(\begin{array}{c}
i_{a} \\
i_{b}
\end{array}\right)
$$

which yields

$$
\begin{aligned}
\tau_{e}= & -K i_{a} \sin \left(n_{p} \theta\right)+K i_{b} \cos \left(n_{p} \theta\right) \\
& +n_{p} L_{2}\left(\left(-i_{a}^{2}+i_{b}^{2}\right) \sin \left(2 n_{p} \theta\right)+2 i_{a} i_{b} \cos \left(2 n_{p} \theta\right)\right)(8)
\end{aligned}
$$

where $K=n_{P} \Psi_{0}$ is the torque constant (and also the back-EMF constant).

\subsection{Model in the rotating frame $d-q$ :}

The model can be transformed using Park's transformation Park [1929],

$$
\left(\begin{array}{l}
\Psi_{d} \\
\Psi_{q}
\end{array}\right)=U(\theta)\left(\begin{array}{l}
\Psi_{a} \\
\Psi_{b}
\end{array}\right)
$$

where

$$
U(\theta)=\left(\begin{array}{cc}
\cos \left(n_{p} \theta\right) & \sin \left(n_{p} \theta\right) \\
-\sin \left(n_{p} \theta\right) & \cos \left(n_{p} \theta\right)
\end{array}\right)
$$

The inverse transformation is

$$
\left(\begin{array}{c}
\Psi_{a} \\
\Psi_{b}
\end{array}\right)=U^{T}(\theta)\left(\begin{array}{l}
\Psi_{d} \\
\Psi_{q}
\end{array}\right)
$$

Differentiating (11)

$$
\frac{d}{d t}\left(\begin{array}{l}
\Psi_{a} \\
\Psi_{b}
\end{array}\right)=U^{T}(\theta) \frac{d}{d t}\left(\begin{array}{l}
\Psi_{d} \\
\Psi_{q}
\end{array}\right)+\omega \frac{\partial U^{T}(\theta)}{\partial \theta}\left(\begin{array}{c}
\Psi_{d} \\
\Psi_{q}
\end{array}\right)
$$

where

$$
\frac{\partial U(\theta)}{\partial \theta}=n_{P}\left(\begin{array}{cc}
-\sin \left(n_{p} \theta\right) & \cos \left(n_{p} \theta\right) \\
-\cos \left(n_{p} \theta\right) & -\sin \left(n_{p} \theta\right)
\end{array}\right)
$$

Therefore 


$$
\begin{aligned}
\frac{d}{d t}\left(\begin{array}{c}
\Psi_{d} \\
\Psi_{q}
\end{array}\right) & =U(\theta) \frac{d}{d t}\left(\begin{array}{l}
\Psi_{a} \\
\Psi_{b}
\end{array}\right)-\omega U(\theta) \frac{\partial U^{T}(\theta)}{\partial \theta}\left(\begin{array}{l}
\Psi_{d} \\
\Psi_{q}
\end{array}\right) \\
& =U(\theta)\left(\begin{array}{c}
v_{a}-R i_{a} \\
v_{b}-R i_{b}
\end{array}\right)-n_{p} \omega\left(\begin{array}{cc}
0 & -1 \\
1 & 0
\end{array}\right)\left(\begin{array}{c}
\Psi_{d} \\
\Psi_{q}
\end{array}\right) \\
& =\left(\begin{array}{c}
v_{d}-R i_{d}+n_{p} \omega \Psi_{q} \\
v_{q}-R i_{q}-n_{p} \omega \Psi_{d}
\end{array}\right)
\end{aligned}
$$

while

$$
\begin{aligned}
\left(\begin{array}{c}
\Psi_{d} \\
\Psi_{q}
\end{array}\right) & =U(\theta) L(\theta) U^{T}(\theta)\left(\begin{array}{c}
i_{d} \\
i_{q}
\end{array}\right)+\Psi_{0} U(\theta) D(\theta) \\
& =L(0)\left(\begin{array}{c}
i_{d} \\
i_{q}
\end{array}\right)+\left(\begin{array}{c}
\Psi_{0} \\
0
\end{array}\right)
\end{aligned}
$$

where

$$
L(0)=\left(\begin{array}{cc}
L_{0}+L_{2} & 0 \\
0 & L_{0}-L_{2} \\
&
\end{array}\right)
$$

The $D Q$ model of the hybrid PMSM becomes

$$
\begin{aligned}
& L_{d} \frac{d i_{d}}{d t}=v_{d}-R i_{d}+n_{p} \omega L_{q} i_{q} \\
& L_{q} \frac{d i_{q}}{d t}=v_{q}-R i_{q}-n_{p} \omega L_{d} i_{d}-K \omega
\end{aligned}
$$

where

$$
L_{d}=L_{0}+L_{2}, \quad L_{q}=L_{0}-L_{2}
$$

The torque is

$$
\begin{aligned}
& \tau_{e}=\Psi_{0}\left(i_{d} i_{q}\right) U(\theta) \frac{\partial D(\theta)}{\partial \theta} \\
& +\frac{1}{2}\left(\begin{array}{ll}
i_{d} & i_{q}
\end{array}\right) U(\theta) \frac{\partial L(\theta)}{\partial \theta} U^{T}(\theta)\left(\begin{array}{l}
i_{d} \\
i_{q}
\end{array}\right)
\end{aligned}
$$

After simplifications

$$
\tau_{e}=K i_{q}+n_{p}\left(L_{d}-L_{q}\right) i_{d} i_{q}
$$

The second term in the torque equation is the reluctance torque. In some permanent magnet motors, the torque is small enough that one can assume $L_{d}=L_{q}$. Conversely, a synchronous reluctance motor corresponds to $K=0$. The motor model considered here encompasses all these cases.

The $d-q$ transformation is advantageous because it results in constant voltages and currents at constant speed (instead of the high-frequency phase variables). However, the $d-q$ transformation requires a position measurement that is aligned with the $d$ axis. For systems using an incremental encoder, this means that the system must be initialized so that the back-emf term $K \omega$ only shows up in the $q$ axis, i.e., so that the axis of the PM is aligned with phase $a$ when $\theta=0$.

\subsection{Model in the rotating frame with position offset $f-g$ :}

Consider now the effect of a position offset, so that the actual position is $\delta$ when the measured value is 0 . The $d q$ transformation is applied to the variables using the measured angle, and the transformed variables are labelled $f g$ instead of $d q$. This means that the variables are transformed into an $f-g$ frame of reference rotating at the same speed as the $d-q$ frame, but with some offset angle $\delta$, and

$$
\begin{aligned}
\left(\begin{array}{l}
\Psi_{f} \\
\Psi_{g}
\end{array}\right) & =U(\theta+\delta)\left(\begin{array}{l}
\Psi_{a} \\
\Psi_{b}
\end{array}\right) \\
& =U(\delta)\left(\begin{array}{l}
\Psi_{d} \\
\Psi_{q}
\end{array}\right)
\end{aligned}
$$

Using the same derivations as for the $d-q$ transformation

$$
\frac{d}{d t}\left(\begin{array}{c}
\Psi_{f} \\
\Psi_{g}
\end{array}\right)=\left(\begin{array}{c}
v_{f}-R i_{f}+n_{p} \omega \Psi_{g} \\
v_{g}-R i_{g}-n_{p} \omega \Psi_{f}
\end{array}\right)
$$

while

$$
\begin{aligned}
\left(\begin{array}{l}
\Psi_{f} \\
\Psi_{g}
\end{array}\right) & =U(\delta)\left(\begin{array}{c}
L_{d} i_{d}+\Psi_{0} \\
L_{q} i_{q}
\end{array}\right) \\
& =U(\delta)\left(\begin{array}{cc}
L_{d} & 0 \\
0 & L_{q}
\end{array}\right) U^{T}(\delta)\left(\begin{array}{c}
i_{f} \\
i_{g}
\end{array}\right)+\Psi_{0}\left(\begin{array}{c}
\cos \left(n_{p} \delta\right) \\
-\sin \left(n_{p} \delta\right)
\end{array}\right) \\
& =L(-\delta)\left(\begin{array}{c}
i_{f} \\
i_{g}
\end{array}\right)+\Psi_{0} D(-\delta)
\end{aligned}
$$

It follows that the electrical equations of the $f-g$ model of the motor are

$$
\frac{d}{d t}\left(\begin{array}{c}
i_{f} \\
i_{g}
\end{array}\right)=L^{-1}(-\delta)\left(\begin{array}{c}
v_{f}-R i_{f}+n_{p} \omega \Psi_{g} \\
v_{g}-R i_{g}-n_{p} \omega \Psi_{f}
\end{array}\right)
$$

and the torque is

$$
\begin{aligned}
\tau_{e} & =K\left(i_{f} \sin \left(n_{p} \delta\right)+i_{g} \cos \left(n_{p} \delta\right)\right) \\
& +n_{p}\left(L_{d}-L_{q}\right)\left(\frac{1}{2} \sin \left(2 n_{p} \delta\right)\left(i_{f}^{2}-i_{g}^{2}\right)+\cos \left(2 n_{p} \delta\right) i_{f} i_{g}\right)
\end{aligned}
$$

\section{IDENTIFICATION OF THE PARAMETERS}

In this section, a method is proposed for the identification of the machine parameters, including the offset angle. The least-squares algorithm is at the heart of the identification procedure (Blauch et al. [1993]). Consider the equation

$$
y[n]=W^{T}[n] P_{\text {nom }}
$$

where $y$ is the output vector, $W$ is the regressor matrix, and $P_{\text {nom }}$ is the nominal (unknown) parameter vector. Given measurements of $y$ and $W$, the objective is to determine $P$, an estimate of the nominal parameter vector $P_{n o m}$. The error equation is formed by subtracting the output from the estimated output. By definition, the residual error $R_{e}$ is equal to the sum of the norm squared error over an interval $\left[N_{0}, N_{1}\right]$, and the least-squares estimate minimizes the residual error.

\subsection{Identification algorithm without offset}

At steady-state, the $d-q$ variables are constant. With $\delta=0$, it follows that

$$
\left(\begin{array}{l}
v_{d} \\
v_{q}
\end{array}\right)=\left(\begin{array}{cccc}
i_{d} & 0 & -n_{p} \omega i_{q} & 0 \\
i_{q} & n_{p} \omega i_{d} & 0 & K \omega
\end{array}\right)\left(\begin{array}{l}
R \\
L_{q} \\
L_{d} \\
K
\end{array}\right)
$$

Equation (26) is applicable with 


$$
\begin{aligned}
y[n] & =\left(\begin{array}{l}
v_{d}[n] \\
v_{q}[n]
\end{array}\right) \\
W[n] & =\left(\begin{array}{cc}
i_{d}[n] & i_{q}[n] \\
-n_{p} \omega[n] i_{q}[n] & 0 \\
0 & n_{p} \omega i_{d}[n] \\
0 & \omega[n]
\end{array}\right) \\
P_{\text {nom }} & =\left(\begin{array}{lll}
R & L_{d} & L_{q} K
\end{array}\right)^{T}
\end{aligned}
$$

Then, $L_{0}=\left(L_{d}+L_{q}\right) / 2$ and $L_{2}=\left(L_{d}-L_{q}\right) / 2$.

\subsection{Identification algorithm with offset}

In the same manner, the variables in the $f-g$ frame $(24)$ are related by

$$
\left(\begin{array}{c}
v_{f} \\
v_{g}
\end{array}\right)=R\left(\begin{array}{c}
i_{f} \\
i_{g}
\end{array}\right)+n_{p} \omega\left(\begin{array}{c}
-\Psi_{f} \\
\Psi_{g}
\end{array}\right)
$$

where

$$
\begin{aligned}
\left(\begin{array}{l}
\Psi_{f} \\
\Psi_{g}
\end{array}\right)= & \left(\begin{array}{cc}
L_{0}+L_{2} \cos \left(2 n_{p} \delta\right) & -L_{2} \sin \left(2 n_{p} \delta\right) \\
-L_{2} \sin \left(2 n_{p} \delta\right) & L_{0}-L_{2} \cos \left(2 n_{p} \delta\right)
\end{array}\right)\left(\begin{array}{c}
i_{f} \\
i_{g}
\end{array}\right) \\
& +K \omega\left(\begin{array}{c}
\sin \left(n_{p} \delta\right) \\
\cos \left(n_{p} \delta\right)
\end{array}\right)
\end{aligned}
$$

Equation (26) is applicable with

$$
\begin{aligned}
& y[n]=\left(\begin{array}{l}
v_{f}[n] \\
v_{g}[n]
\end{array}\right) \\
& W[n]=\left(\begin{array}{cc}
i_{f}[n] & i_{g}[n] \\
n_{p} \omega[n] i_{f}[n] & -n_{p} \omega[n] i_{g}[n] \\
-n_{p} \omega[n] i_{g}[n] & 0 \\
0 & n_{p} \omega i_{f}[n] \\
\omega[n] & 0 \\
0 & \omega[n]
\end{array}\right) \\
& P_{\text {nom }}=\left(R L_{1} \sin \left(2 n_{p} \delta\right) L_{0}-L_{1} \cos \left(2 n_{p} \delta\right) \ldots\right. \\
& \left.L_{0}+L_{1} \cos \left(2 n_{p} \delta\right) K \sin \left(n_{p} \delta\right) K \cos \left(n_{p} \delta\right)\right)^{T}
\end{aligned}
$$

From the estimated vector $P$, estimates of the original parameters can be deduced using $R=p_{1}, L_{0}=\left(p_{3}+p_{4}\right) / 2$, $K=\sqrt{p_{5}^{2}+p_{6}^{2}}, L_{2}=\left(p_{5}^{2}+p_{6}^{2}\right) p_{2} /\left(2 p_{5} p_{6}\right)$, and

$$
\delta=\left\{\begin{array}{ccc}
\arctan \left(\frac{p_{5}}{p_{6}}\right) / n_{p} & \text { if } \quad \hat{c}>0 \\
\pi / 2 n_{p} & \text { if } \quad \hat{s}=1 \\
-\pi / 2 n_{p} & \text { if } \quad \hat{s}=-1 \\
\left(\arctan \left(\frac{p_{5}}{p_{6}}\right)-\pi\right) / n_{p} & \text { if } \quad \hat{c}<0 \\
& \text { and } \quad \hat{s}>0 \\
\left(\arctan \left(\frac{p_{5}}{p_{6}}\right)+\pi\right) / n_{p} & \text { if } & \hat{c}<0 \\
& \text { and } & \hat{s}<0
\end{array}\right.
$$

where $\hat{c}=p_{6} / \sqrt{p_{5}^{2}+p_{6}^{2}}, \hat{s}=p_{5} / \sqrt{p_{5}^{2}+p_{6}^{2}}$ are estimates of $\cos \left(n_{p} \delta\right)$ and $\sin \left(n_{p} \delta\right)$, respectively.

\section{EXPERIMENTAL RESULTS}

Experiments were performed by applying various voltages $v_{f}$ and $v_{g}$. Due to the open-loop stability of the motor, the speed converged even with a position offset. Once the velocity was (approximately) constant, the average values of the currents and of the velocity were computed over a small time interval, yielding one data point for the leastsquares algorithm. Various values of $v_{f}$ and $v_{g}$ were applied to the motor to obtain velocities covering a large range. Multiple data points were thereby obtained for the leastsquares algorithm, from which estimates of the parameters were computed.

To evaluate the results, two cases are discussed. In the first subsection, $f-g$ identification is performed using the encoder with an offset $\delta$. In the second subsection, the encoder is initialized by applying a DC current to winding $a$ and $d-q$ identification is performed.

\subsection{Identification with position offset}

To show the performance of the offset estimator, identification was performed with and without the parameter $\delta$. Fig. 3 shows the output vector from (26) as a function of $\omega$, which is the same in both cases. The components of the output vector are the voltages $v_{f}$ and $v_{g}$, whose jagged appearance is due to the fact that multiple values of the voltages can yield the same velocity, and multiple values were deliberately applied to increase the richness of the data. The green curve shows the fit from the leastsquares algorithm (i.e., $W^{T}[n] P$, where $P$ is the leastsquares estimate of the parameters) when the offset is not estimated. The red curve shows the least-squares fit when the offset is estimated. The match of the least-squares fit with the data on the figure is found to be very good when the offset is included, but not otherwise.

The estimated values are given in Table 1 . Case 1 is when the offset is not included, and case 2 when it is. The electrical parameters and the back-emf will be compared to the parameters identified in the next section using the $d-q$ procedure. The angle estimated in the experiment is $\delta=\frac{-1.08}{50} \mathrm{rad}=-1.24^{\circ}$, or 62 electrical degrees.

\subsection{Identification with encoder initialization}

When the encoder is initially calibrated using a DC current, the least-squares algorithm is applied in the $d-q$ frame using equations (27). The results are shown Fig. 4. Similarly to the previous section, the output vector from
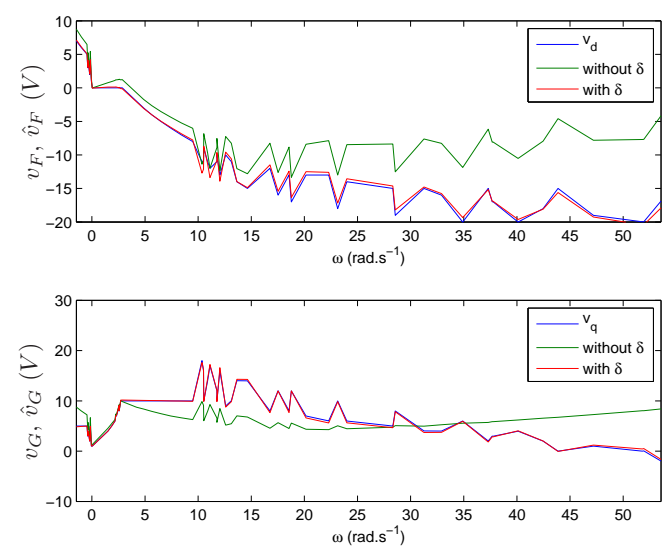

Fig. 3. Representation of the identification of the electrical parameters and the calibrage offset in the $f-g$ frame 

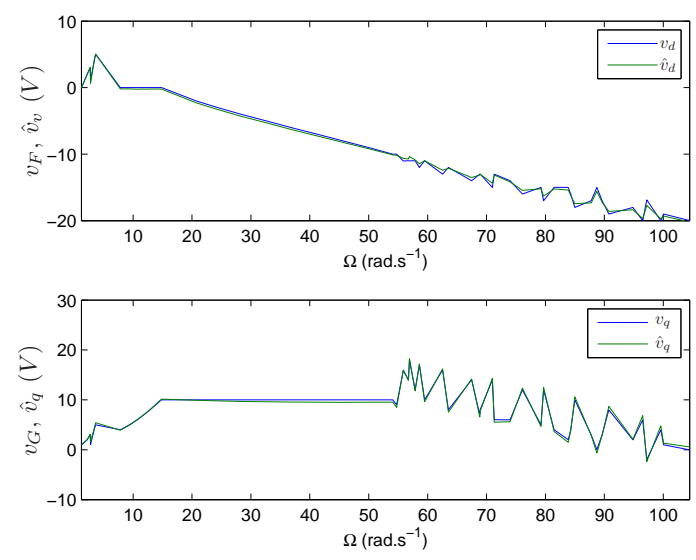

Fig. 4. Representation of the identification of the electrical parameters in the $d-q$ frame

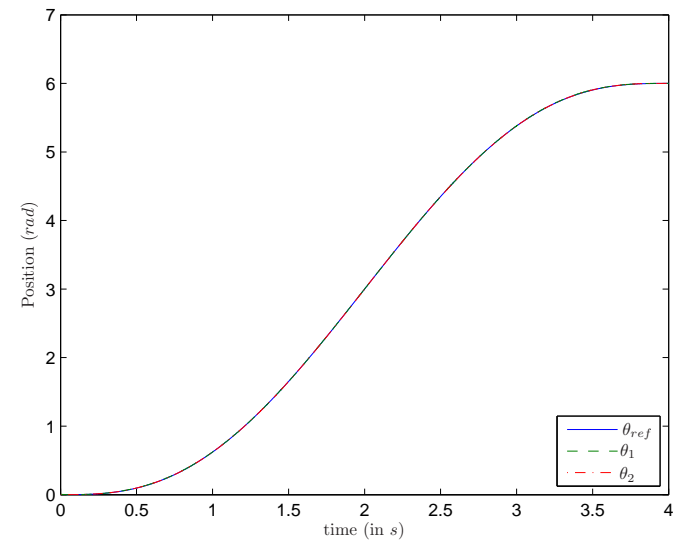

Fig. 5. Trajectory tracking (Slow experimentation)

(26) is shown as a function of $\omega$, as well as the fit from the least-squares algorithm. The least-squares fit is very good. The results are shown in Table 1 as case 3 . Compared to case 2 , the estimates are very close. The small variation between the parameters may originate from the fact that the experiments are different. Indeed, Fig 4 shows that the velocity reached by the motor for the same input voltage is bigger when the position is initialized.

\section{CLOSED LOOP PERFORMANCES}

The estimated parameters were also used in closed-loop control experiments. The control law used in this section relied on the flatness of the model and high-order sliding modes (see Nollet et al. [2008]). The control law and the trajectory to be tracked are the same as in Nollet et al. [2008], but with the inductance $L$ replaced by $L_{d}$ and $L_{q}$

\begin{tabular}{|c|c|c|c|}
\hline \hline Parameters & Case 1 & Case 2 & Case 3 \\
\hline$R(\Omega)$ & 3.69 & 2.80 & 2.83 \\
$L_{0}(m H)$ & 10.2 & 9.8 & 10.7 \\
$L_{2}(m H)$ & -0.36 & -0.59 & -0.33 \\
$K\left(\right.$ Nm. $\left.^{-1}\right)$ & 0.088 & 0.29 & 0.27 \\
$\delta\left(\right.$ rad.s $\left.^{-1}\right)$ & $X$ & $-21.7 .10^{-3}$ & $\mathrm{X}$ \\
\hline
\end{tabular}

Table 1. Results of the parameter estimation in the $d-q$ frame. where applicable. We present the results of experiments at two different speeds. For both cases, the tracking is shown for the angle calibrated using the estimated angle $\delta$, and using an initialization with a DC current $i_{a}$.

The desired position in the "slow" experiment goes from 0 to $6 \mathrm{rad}$ in 4 seconds, following a trajectory based on a Bernstein polynomial interpolation equation of degree 5 . On Fig 5, the following variables are plotted: the desired position $\theta_{\text {ref }}$ is shown in blue, the position in the case where the estimated $\delta$ is used (case 1) is shown in green, and the position in the case where the position is initialized using $i_{a}$ (case 2 ) is shown in red. Fig 6 shows the respective errors. One finds that there is no major difference between the two solutions.

For the second experiment, the position moves from 0 to $60 \mathrm{rad}$ in 4 seconds. The results are plotted Fig. 7 and 8 and show that, at higher speed, both solutions gives comparable results.

\section{CONCLUSIONS}

In this paper, a method was proposed to estimate jointly the PMSM parameters and the initial offset angle of an incremental encoder. Special cases of the hybrid PMSM model include pure PM motors and synchronous reluctance motors. Although initialization of an encoder can be easily performed by applying a current to one phase of the motor, the method assumes that the load and friction torques are sufficiently small so that the position reaches the desired position. The proposed method does not make such an assumption and has the additional benefit of estimating not only the offset angle but also the machine's electrical parameters. Such results could be used for the tuning of the control law, or for failure detection. The use of online estimation methods using the algorithms presented in Delpoux and Floquet [2011] is also being investigated. As parameters may vary over time, such methods would make it possible to update the model parameters during real-time operation.

\section{REFERENCES}

A. J. Blauch, M. Bodson, and J. Chiasson. High-speed parameter estimation of stepper motors. Control Systems

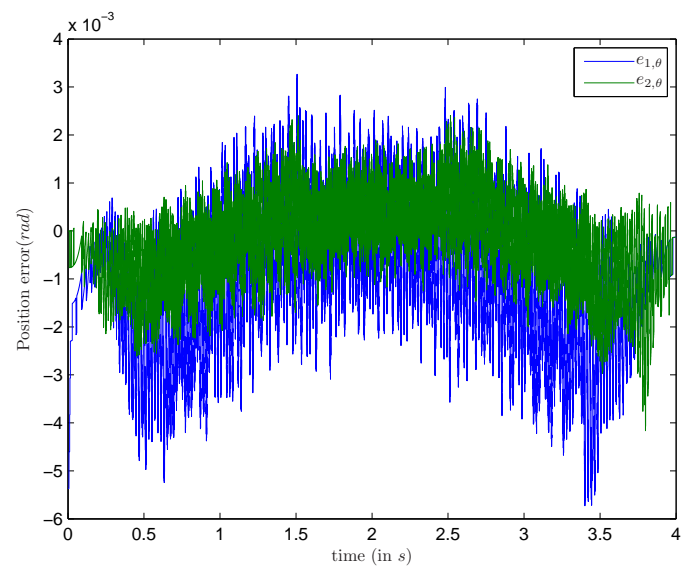

Fig. 6. Tracking error (Slow experimentation) 


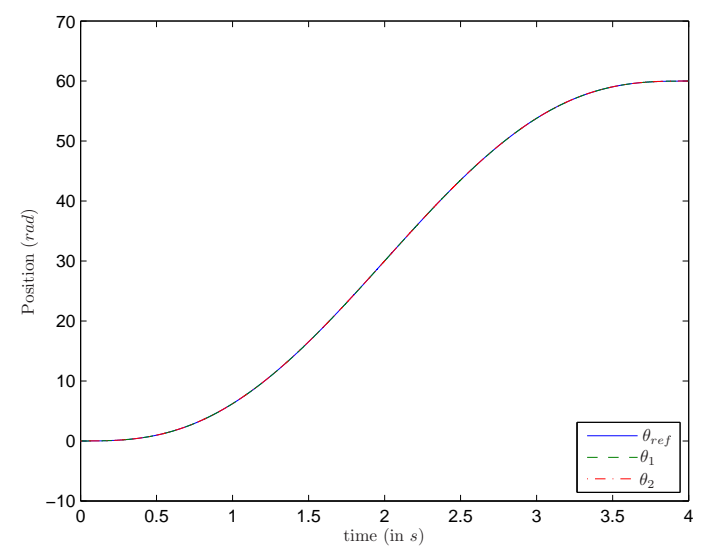

Fig. 7. Trajectory tracking (Fast experimentation)

Technology, IEEE Transactions on, 1(4):270 -279, dec 1993. ISSN 1063-6536. doi: 10.1109/87.260272.

R. Delpoux and T. Floquet. On-line parameter estimation of a magnetic bearing. In Control Automation (MED), 2011 19th Mediterranean Conference on, pages 388 393, june 2011. doi: 10.1109/MED.2011.5983117.

C. Goeldel. Contribution à la modélisation, à l'alimentation et à la commande des moteurs pas à pas, $\mathrm{PhD}$ dissertation, Institut National Polytechnique de Lorraine, 1984.

D. H. Jung and I. J. Ha. An efficient method for identifying the initial position of a pmsm with an incremental encoder. Industrial Electronics, IEEE Transactions on, 45(4):682 -685, aug 1998. ISSN 0278-0046. doi: $10.1109 / 41.704901$.

H. Kim and R.D. Lorenz. Improved current regulators for ipm machine drives using on-line parameter estimation. In Industry Applications Conference, 2002. 37th IAS Annual Meeting. Conference Record of the, volume 1, pages 86 - 91 vol.1, 2002. doi: 10.1109/IAS.2002.1044071.

M. Konghirun. Automatic offset calibration of quadrature encoder pulse sensor for vector controlled drive of permanent magnet synchronous motors. In TENCON 2005 2005 IEEE Region 10, pages 1 -5, nov. 2005. doi: 10.1109/TENCON.2005.301305.

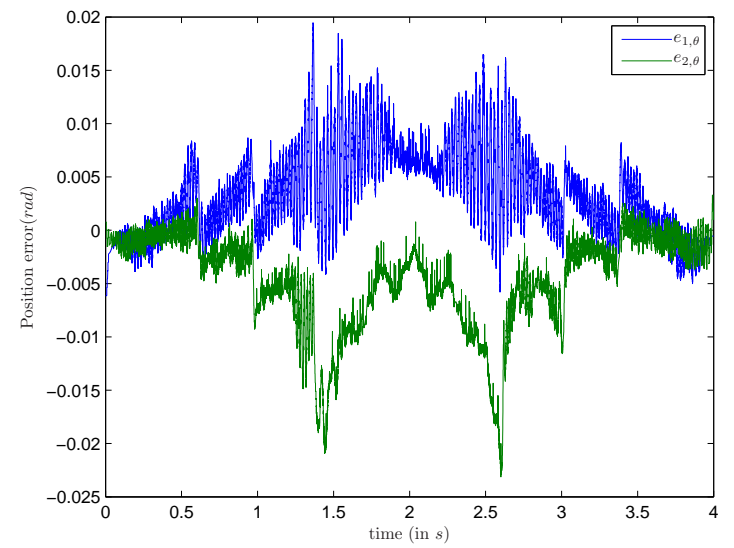

Fig. 8. Tracking error (Fast experimentation)
B. Nahid Mobarakeh, F. Meibody-Tabar, and F.M. Sargos. On-line identification of pmsm electrical parameters based on decoupling control. In Industry $A p$ plications Conference, 2001. Thirty-Sixth IAS Annual Meeting. Conference Record of the 2001 IEEE, volume 1, pages $266-273$ vol.1, sep-4 oct 2001. doi: 10.1109/IAS.2001.955421.

F. Nollet, T. Floquet, and W. Perruquetti. Observerbased second order sliding mode control laws for stepper motors. Control Engineering Practice, 16 (4):429 - 443, 2008. ISSN 0967-0661. doi: 10.1016/j.conengprac.2007.05.008.

R. H. Park. Two-reaction theory of synchronous machines generalized method of analysis-part i. American Institute of Electrical Engineers, Transactions of the, 48(3): $716-727$, july 1929. ISSN 0096-3860. doi: 10.1109/TAIEE.1929.5055275.

P. Segarich, M. Carpaneto, M. Marchesoni, A. De Cian, C. Ladisa, and F. Soso. A new procedure to detect rotor position during startup in medium voltage synchronous motor drives with incremental encoder. In Power Electronics and Applications, 2009. EPE'09. 13th European Conference on, pages $1-9$, sept. 2009.

Naresh K. Sinha, A. R. Elliott, and Richard C. S. Wong. A realistic mathematical model for permanentmagnet stepping motors. Industrial Electronics and Control Instrumentation, IEEE Transactions on, IECI21(3):168 -173, aug. 1974. ISSN 0018-9421. doi: 10.1109/TIECI.1974.351139. 